\title{
Luminex Crossmatch as a Successful Algorithm for Post-Transplant Follow-up in Developing Nations-A Study of 169 Consecutive Samples
}

Mahendra Narain Mishra* and Vandana Lal

Belfast City Hospital, Antrim, UK

"Corresponding author: Mahendra Narain Mishra, MBBS, MD, ESHI Diploma, Belfast City Hospital, Antrim, UK, Tel: +44 2890329241; E-mail: mnmishra@hotmail.com

Received date: April 27, 2016; Accepted date: May 25, 2016; Published date: June 1, 2016

Copyright: (C) 2016 Mishra MN, et al. This is an open-access article distributed under the terms of the Creative Commons Attribution License, which permits unrestricted use, distribution, and reproduction in any medium, provided the original author and source are credited.

\begin{abstract}
Background: Since 2014 Luminex crossmatch (LXM) is being performed regularly in India for post-transplant management of renal allograft recipients.

Aim: This study was planned to evaluate the possible role of the LXM in the post-transplant setting for the detection and characterization of HLA-specific $\operatorname{lgG}$ antibodies and to correlate with allograft biopsy and renal function.

Methods: A retrospective study was performed on 169 consecutive LXM performed at a single center to investigate suspected allograft dysfunction $(n=147)$ and for monitoring DSA in normally functioning allografts $(n=22)$. A total of 116 biopsies (including 11 repeats) and 40 panel reactive antibody (PRA) screen tests were performed.

Results: Donor specific antibodies (DSA) were detected in 81 samples (47.9\%): 7 recipients had HLA class I DSA alone, 56 had only HLA class II DSA and 18 recipients had both. First time biopsies were categorized as: negative for rejection $(n=30)$, antibody mediated rejection $(n=32)$, acute cellular rejection $(n=23)$, chronic rejection $(n=15)$ and dual morphology $(n=5)$. LXM was positive in $44 / 75$ biopsies with rejection and transiently raised in $11 / 30$ recipients who showed no evidence of rejection. On the basis of the LXM results biopsy was not performed in 42 recipients who showed improved renal function 15 recipients with non-specific findings on first biopsy.
\end{abstract}

Conclusion: The study suggests that LXM is useful and economically viable algorithm for the detection of antibodies in the post-transplant work up of renal allograft recipients in developing nations.

Keywords: Luminex crossmatch; Biopsy; Renal allograft; Posttransplant

\section{Introduction}

Evaluation of renal function, immunosuppressant levels, combinations of imaging techniques such as ultrasound or DTPA scans are performed prior to resorting to allograft biopsy, which although extremely safe, is an invasive procedure. Donor-specific HLA antibodies have been implicated in hyperacute and acute rejection episodes as well as chronic renal allograft nephropathy. The posttransplantation group at the Rome Consensus Conference suggested guidelines for the frequency of DSA testing by solid phase immunoassay and biopsy, according to the risk category of the recipients [1].

Luminex crossmatch (LXM) is essentially is a solid phase immunoassay - a qualitative test which detects only antibodies directed against HLA-A, -B and DRB1 [2]. Theoretically, it is a real (physical) crossmatch in which recipient sera are tested against a lysate derived from donor peripheral blood mononuclear cells. The HLA molecules are isolated from these mononuclear cells by treatment with beads coated with anti-human HLA class I or II monoclonal antibodies [3]. Therefore, hypothetically, it can detect donor specific antibodies without the requirement for donor HLA typing. While some centers report the LXM test result as positive or negative: others, including our center, reports the results in terms of MFI (mean fluorescence intensity) to allow the quantitative grading of antibody reactivity and for monitoring the efficacy of immunosuppression. The low cost of the LXM, no requirement for viable cells, and the ability to store donor lysate for future use, coupled with the ease of interpretation, has made it more popular in India than the single antigen bead (SAB) assay which is regarded by many as being far too sensitive. The clinical relevance of donor specific antibodies identified only using SAB but negative by other methods is questionable [4,5]. In spite of its inability to detect donor specific antibodies directed against HLA C, DQ and DP antigens, the test could prove useful in countries where extensive antibody work up is not possible due to financial constraints. Organ Donor and Transplantation United Kingdom allocates deceased donor kidneys on basis of the matching at HLA- ABDR loci, emphasizing that these are the most relevant antigens for kidney transplantation. In India, many private sector hospitals do not perform HLA typing for biologically unrelated donors and practically no center carries out typing for all six HLA loci (A,B,C,DR,DQ, and DP) for pre-transplant work up. If the HLA typing results for these loci for recipient and donor are not available, the identification of antibodies detected against these antigens will have limited value. Expression of HLA-C, DQ and DP is lower on human cells and on phenotype beads, compared to $\mathrm{SAB}$, which are relatively antigen dense and are therefore 
likely to detect antibodies in titers that may not be clinically relevant. Tests using $\mathrm{SAB}$ are therefore more likely to be positive and may be clinically less significant [4]. Flow cytometry crossmatch (FCXM) is useful for pre-transplant work up, especially in re-transplants. A B-cell positive FCXM is tricky to interpret for dual positivity may imply only presence of class I antibodies [6].

There are only a handful of papers that have evaluated the role of the LXM and all are unanimous in the opinion that HLA class II positivity in this test has a limited correlation with acute rejection and graft failure. Billen, et al. have suggested that the LXM positive for class II DSA does not contribute towards graft failure and that although class I DSA is not associated with an increase in acute rejection, it may adversely impact long-term graft survival [7]. Huh, et al. reported a higher incidence of biopsy proven acute cellular rejection in a study on 55 recipients with a PRA $>20 \%$ and a positive LXM [8]. LXM positivity for class II DSA has not been reported to be associated with antibody mediated rejection (AMR) so far and the authors who reported that the risk was proportionate to the semi-quantitative Class I DSA MFI [3].

\section{Materials and Methods}

This study was performed over a 25 month period from September 2013 to October 2015 in the HLA department of the National Reference Laboratory of Dr. Lal Path Labs Ltd. New Delhi which provides laboratory support to many renal transplant centers in India and neighboring countries. Approval of the institutional ethical committee and informed consent of all recipients and their donors was obtained. Details of renal function, history and biopsy findings were obtained from the referring transplant centers.

The primary aim of this study was to evaluate the possible role of LXM in the post-transplant setting for detection and characterization of HLA-specific IgG antibodies and to correlate with biopsy data and renal function tests, with the objective of using the LXM as a low cost algorithm in India where extensive antibody work up is not possible using SAB assays. No additional budget had been allocated for this study so the number of PRA screens and antibody definition tests were as per prescription and the authors were unable to perform any further tests.

\section{Recipients}

LXM was performed on 169 samples obtained from renal receipts transplanted between 14 days and 10 years (median 1.5 years) before giving the sample.A pre-transplant LXM was carried out for 41 recipients as the test has become available widely in India since 2014. Sensitization history -Twelve recipients had a previous transplant, 25 had a history of one to three pregnancies and 43 had received one or more transfusions. However, all recipients were potentially sensitized as they were all allograft recipients.

Allograft biopsy was performed on 105 recipients, of whom 11 required a second follow-up biopsy. Staining for C4d was performed on 65 biopsies which included 36 recipients with AMR and 30 without AMR. Pre-transplant DSA tests were performed on more than 700 recipients (unpublished data) but for this group, information for only 41 recipients is available, because many of them were transplanted before this test became available in India. Thirty recipients were tested more than once by LXM following a biopsy or for gauging the response to treatment.
PRA screening for the detection of HLA class I and II antibodies was performed by a pooled assay using LMX Life codes screen Deluxe kits (Immucor, USA) as per manufacturer's instructions for 40 samples to confirm the presence of DSA, including 20 samples with high background as shown in Table 2 .

\begin{tabular}{|l|l|}
\hline Parameter & Numbers \\
\hline Recipient age & $19-65$ (M 39.4) \\
\hline Donor age & $24-67$ (M 46.5) \\
\hline Relationship & 52 \\
\hline Parent & 12 \\
\hline Sibling & 42 \\
\hline Spouse & 10 \\
\hline Offspring & 12 \\
\hline Non 1st degree relative & 28 \\
\hline Unrelated & 8 \\
\hline Paired exchange & 5 \\
\hline Deceased donor transplant & \\
\hline
\end{tabular}

Table 1: Recipient-donor profile for 169 allograft recipients.

CDC B and T cell crossmatching was performed for 26 samples on $\mathrm{B}$ and $\mathrm{T}$ cells isolated using nylon wool from peripheral blood mononuclear cells using the Extended NIH method [9]. Dithiothretiol treated serum was used to detect alloreactive antibodies and untreated serum was tested to detect IgM auto antibodies. Serum was tested in doubling dilutions up to a titer of 16 to allow for detection of the prozone effect. This was done along with LXM before the latter gained acceptance among nephrologists.

\section{Panel reactive antibody specification}

Five samples were tested upon request by the referring physicians. Single Antigen Bead assay was used to test samples from four recipients during the course of their management. Subsequently, the LXM was used in their immediate post-transplant management which culminated in their successful transplantation.

Luminex cross match was carried out using DSA Life Codes Donor specific antibody kits (Immucor, USA) as described by Huh et al. with the modification that we did not include a lysate control for each sample, but was an integral part of each run. Results were interpreted using Quicktype software initially and later using Match IT! [3]. The lysate control is used to assess the binding of monoclonal antibodies to HLA-class I and II molecules. The laboratory has performed more than 2000 LXM tests and participates regularly with a satisfactory performance in the College of American Pathologists external proficiency testing survey since 2014 [10].

A LXM result was considered positive if the MFI was $>500$ and if two or more of control beads were positive leading to a score $\geq 2$. A positive LXM result with negative PRA screen and normal histology on two consecutive biopsies was considered to be a false positive result. All negative results were taken as true negative and were correlated with renal function, previous LXM results and biopsy findings where 
Page 3 of 5

available. The MFI does not automatically correlate well with antibody titers because it may be affected by several factors including antibody concentration in the serum, and the density, conformation and orientation of the antigen on the bead, as well as by the antibody avidity toward the respective antigen [6].

High background was observed in nearly 20 samples (11.8\%) and persisted in six cases even with a repeat sample. We did not use Seraclean (Immucor, USA) because we found that it reduced but did not remove the background completely in ten samples tested during validation and hence was not used in the study due to its high cost and inability to completely remove the high background. Fresh samples obtained from the same six recipients were also tested by the pooled assay method to confirm the results. Allograft biopsies were performed for 105/169 recipients but not in others, because the renal function was normal $(n=22)$ or because impaired function reversed readily with treatment $(n=42)$. Staining for C4d was performed by immunofluorescence. Evaluation of the biopsies was carried out in accordance with Banff criteria [11].

\section{Immunosuppression}

The choice of regimen varied between centers but most included a combination of Tacrolimus, Prednisolone and Mycophenolate, with Rituximab and ATG for suspected acute rejection episodes. Some recipients with pretransplant DSA were administered plasmapheresis, Rituximab and Calcineurin inhibitors prior to the transplant. Recipients with biopsy proven AMR were administered corticosteroids together with a combination of plasmapheresis, intravenous Immunoglobulin, Rituximab and/or anti thymocyte globulin. Statistical analysis of the accrued data was performed by using Graph Pad software www.graphpad.com/quickcalcs and $\mathrm{p}$ values were calculated.

\section{Results}

The mean age of recipients' was 39.2 years (range 19-65 years) with males accounting for $70 \%$. The mean donor age was 46.5 years (range 24-67 years) with a female preponderance (62.5\%). All except five recipients had living donors. The donor-recipient profile is depicted in Table 1.

Table 2 shows the recipient gender, and Luminex crossmatch DSA results, PRA screen results, and biopsy findings that have been categorized under six different types. Recipients were categorized according to the biopsy classification with a separate category for those not biopsied. There were eight false positive DSA- all had class II IgG which was confirmed by the PRA screen and were detected in the nonbiopsied recipients, and those with non-specific findings.

Three false positive samples had a high background when tested, therefore making it necessary to closely scrutinize such results. In other samples with high background, the class I MFI was $>500$ but when the reactivity of the background beads was evaluated the score was less than two.

Reactivity against DQB1 antigens was detected in seven samples as shown by high reactivity against DQ enriched beads in the PRA screen and a positive LXM class II results which points to the fact that the test may sometimes detect anti DQB1 reactivity if it is above a certain threshold.

Luminex crossmatch was shown to give to false negative results, evidenced by a high PRA specification in two recipients who went on to develop acute cellular rejection. The lowest MFI value was 67 (negative) with a peak of 11672 for HLA-class I and 15305 for-class II DSA.

\begin{tabular}{|c|c|c|c|c|c|c|c|c|}
\hline \multirow{2}{*}{ Category } & \multirow{2}{*}{$\begin{array}{l}\text { Gender } \\
\text { M:F }\end{array}$} & \multirow{2}{*}{ DSA negative } & \multicolumn{3}{|c|}{ DSA Positive } & \multirow[t]{2}{*}{ PRA screen } & \multirow[t]{2}{*}{ Previous DSA } & \multirow[t]{2}{*}{ False positive } \\
\hline & & & Class I & Class II & Both & & & \\
\hline \multicolumn{9}{|l|}{ I Biopsy not done } \\
\hline $\begin{array}{l}\text { I A Normal renal } \\
\text { function }(n=22)\end{array}$ & 17:05 & 15 & 0 & 7 & 0 & 4 & 4 & 1 \\
\hline $\begin{array}{l}\text { I B Allograft } \\
\text { dysfunction }(n=42)\end{array}$ & $30: 12$ & 23 & 2 & 13 & 4 & 10 & 10 & 3 \\
\hline $\begin{array}{l}\text { Il Biopsy negative for } \\
\operatorname{AR}(n=30)\end{array}$ & \multirow{3}{*}{ 22:08 } & \multirow{3}{*}{19} & \multirow{3}{*}{0} & \multirow{3}{*}{10} & \multirow{3}{*}{1} & \multirow{3}{*}{8} & \multirow{3}{*}{10} & \multirow{3}{*}{4} \\
\hline IIA ATN (10) & & & & & & & & \\
\hline II B Drug toxicity (5) & & & & & & & & \\
\hline IIC Nonspecific* (15) & \multicolumn{8}{|c|}{${ }^{*}$ Included in biopsy negative for rejection but was not possible to assign specific pathology } \\
\hline III ACR $(n=23)$ & 15:08 & 12 & 1 & 9 & 1 & 5 & 5 & 0 \\
\hline IV AMR $(n=32)$ & $20: 12$ & $9\left({ }^{*} 4\right)$ & 4 & 9 & 10 & 9 & 8 & 0 \\
\hline $\begin{array}{l}\text { V Dual morphology } \\
\text { AMR and ACR }(n=5)\end{array}$ & 4:01 & $1^{*}$ & 0 & 3 & 1 & 1 & 2 & 0 \\
\hline $\begin{array}{l}\text { VI Chronic rejection } \\
(n=15)\end{array}$ & 11:04 & 9 & 0 & 5 & 1 & 3 & 2 & 0 \\
\hline
\end{tabular}


Page 4 of 5

\begin{tabular}{|l|l|l|l|l|l|l|l|l|}
\hline Total & $119: 50$ & 88 & 7 & 56 & 18 & 40 & 41 & 8 \\
\hline
\end{tabular}

Table 2: Luminex crossmatch, PRA screen and false positive results in renal allograft recipients. DSA: Donor Specific Antibodies detected by Luminex crossmatch; AR: Acute Rejection; ACR: Acute Cellular Rejection; AMR: Antibody Mediated Rejection; ATN: Acute Tubular Necrosis; ${ }^{*}$ treated patients.

In antibody negative group the incidence of ACR and chronic rejection was higher, but this difference was not statistically significant. The frequency of biopsies negative for rejection was higher in antibody negative recipients and those with dual morphology (ACR and AMR) was higher in the antibody positive group, but neither was statistically significant. AMR frequency in antibody positive assumed statistical significance $(\mathrm{p}=0.031)$ as shown in Table 3 .

\begin{tabular}{|c|c|c|c|}
\hline Parameter & $\begin{array}{l}\text { Antibody } \\
\text { positive } n=81\end{array}$ & $\begin{array}{l}\text { Antibody negative } \\
n=88\end{array}$ & $P$ value \\
\hline Class I positive & 7 & \multirow{3}{*}{ - } & \\
\hline Class II positive & 56 & & \\
\hline Both positive & 18 & & \\
\hline Biopsy not done & 26 & 38 & 0.151 \\
\hline Normal renal function & (7) & (15) & 0.257 \\
\hline Negative for rejection & 11 & 19 & 0.227 \\
\hline Acute cellular rejection & 11 & 12 & 1 \\
\hline Acute AMR & 23 & 9 (treated-4) & 0.0031 \\
\hline AMR and ACR & 4 & 1 (treated) & 0.19 \\
\hline $\begin{array}{l}\text { Chronic Rejection } \\
\text { (all>3 years back) }\end{array}$ & 6 & 9 & 0.59 \\
\hline
\end{tabular}

Table 3: Recipients classified by DSA positivity and clinical/biopsy categories.

\section{Biopsy}

The five categories of recipients classified on basis of histopathological features are shown in tables 2 and 3. Of the recipients with features of chronic rejection five were C4d positive and two had features of thrombotic microangiopathy. Staining for C4d was diffuse positive in 25 biopsies with AMR and focal positive in four biopsies without morphological evidence of AMR.

DSA MFI values in recipients with histology negative for acute rejection was less than 1500 and none of the samples were positive for HLA -class I IgG alone.

\section{Discussion}

This study included renal allograft recipients from multiple centers in Indian subcontinent where treatment is not covered by health insurance and it is not feasible to do extensive SAB work up due to high cost and limited availability of the test until a year back. Luminex crossmatch has become extremely popular in India, is widely accepted and most centers in the country that look upon it as a cost effective substitute. Detection of DSA is useful because of its clarity of interpretation and low false positivity in post-transplant recipients. The authors however reported a high rate of false positive results in pretransplant work up [12]. False positive DSA results were confirmed on the basis of a negative PRA screen in eight recipients which was further confirmed in four of them because they were HLA identical with respect to donor for HLA DR. AMR including a combined picture with ACR, was histologically identified in 37/105 (35.2\%) of biopsies of which 27 were positive for DSA. The actual figures for DSA could have been higher, as five recipients were already in receipt of treatment before a sample was drawn for testing. Some other authors also report AMR in 18-37\% of biopsies [13]. The 2013 meeting on Banff classification has defined criteria for identification C4d negative AMR [11]. Billen, et al. in a study of 165 recipients found that LXM positivity was unlikely to have a higher incidence of AR episodes and opined that class II DSA detected by the LXM had a questionable impact on graft survival [14]. The study shows that Class I DSA alone if detected is likely to true positive false positivity was detected only for class II IgG DSA.

This is the largest series of post-transplant recipients that have been tested by LXM. HLA-class II IgG antibodies were present much more frequently in all categories studied which is not in agreement with other published literature on LXM. Phayphet M, et al. also reported a higher incidence of $\mathrm{AR}$ in recipients with HLA-class II positive antibodies [15]. In those with chronic rejection the incidence of class II DSA was higher than class I DSA, an observation which is similar to that of Grenzi, et al. [16]. The possibility of allografts having subclinical AMR which progressed into a chronic form cannot be ruled out. The retrospective nature of this study with allograft dysfunction as the starting point enabled correlation of histopathological features with LXM results and renal function. It is unlikely that the high incidence of HLA-class II antibodies in this study could be purely coincidental and it will be prudent to assign their role in allograft dysfunction, acute and chronic rejection.

Donor specific HLA antibodies were present in seven recipients with normal renal function, 19 with reversible allograft dysfunction and 13 without histological features of acute rejection which could be due to antigenicity of allografts that may not culminate in rejection. Of the 22 individuals with normal renal function, seven had LXM defined DSA due to accommodation, the mechanism of which cannot be explained entirely [17]. These individuals merit closer DSA monitoring and follow-up studies are required on more individuals with positive DSA prior to labelling the antibodies as harmless.

SAB assay is very sensitive and may show sometimes naturally occurring anti HLA antibodies which may be clinically irrelevant and can be excluded only by further extensive testing which can sometimes lead to aggressive but unnecessary immunosuppression.

It was possible to avoid renal biopsy in 42 recipients with transient allograft dysfunction and repeat biopsies in 15 more with non-specific histopathology reports and who were negative for DSA (Table 2). In this study, clinically relevant antibodies could be detected by LXM in combination with the pooled bead (PRA) assay for some recipients with high test background and /or with a discrepant clinical picture. 
Citation: Mishra MN, Lal V (2016) Luminex Crossmatch as a Successful Algorithm for Post-Transplant Follow-up in Developing Nations-A Study of 169 Consecutive Samples. J Kidney 2: 127. doi:10.4172/2472-1220.1000127

Page 5 of 5

\section{Conclusion}

Luminex crossmatch in combination with a PRA screen is likely to be a useful strategy in developing countries for post-transplant followup of renal allograft recipients due to its lower cost and ease of interpretation.

\section{Acknowledgements}

I am extremely grateful to Dr Kay Poulton for going through the manuscript painstakingly and providing valuable suggestions. I will also like to thank my colleague Jeanie Martin for her valuable inputs in writing the manuscript.

\section{References}

1. Tait BD, Süsal C, Gebel HM, Nickerson PW, Zachary AA, et al. (2013) Consensus guidelines on the testing and clinical management issues associated with HLA and non-HLA antibodies in transplantation. Transplantation 95: 19-47.

2. Guillaume N, Mazouz, H, Piot V, Presne C, Westeel PF (2013) Correlation between Luminex donor-specific crossmatches and levels of donorspecific antibodies in pretransplantation screening. Tissue Antigens 82: $16-20$.

3. Riethmüller S, Ferrari-Lacraz S, Müller MK, Raptis DA, Hadaya K, et al. (2010) Donor-specific antibody levels and three generations of crossmatches to predict antibody-mediated rejection in kidney transplantation. Transplantation 90: 160-167.

4. Süsal C, Ovens J, Mahmoud K, Döhler B, Scherer S, et al. (2011) No association of kidney graft loss with human leukocyte antigen antibodies detected exclusively by sensitive Luminex single-antigen testing: a Collaborative Transplant Study report. Transplantation 91: 883-887.

5. Lachmann N, Todorova K, Schulze H, Schönemann C (2013) Luminex ${ }^{\circ}$ and its applications for solid organ transplantation, hematopoietic stem cell transplantation, and transfusion. Transfus Med Hemother 40: 182-189.
6. Bas le-Bernardet S, Hourmant M, Valentin N, Paitier C, Classe MG. et al. (2003) Identification of the antibodies involved in B-cell crossmatch positivity in renal transplantation. Transplantation 75: 477-482.

7. Billen EV, Christiaans MH, van den Berg-Loonen EM (2009) Clinical relevance of Luminex donor-specific crossmatches: data from 165 renal transplants. Tissue Antigens 74: 205-212.

8. Huh KH, Kim MS, Kim HJ, Joo DJ, Kim BS, et al. (2012) Renal transplantation in sensitized recipients with positive luminex and negative CDC (complement-dependent cytotoxicity) crossmatches. Transpl Int 25: 1131-1137.

9. Gebel HM, Bray RA, Nickerson P (2003) Pre-transplant assessment of donor-reactive, HLA-specific antibodies in renal transplantation: contraindication vs. risk. Am J Transplant 3: 1488-1500.

10. Mishra MN, Lal V (2015) External proficiency test for Luminex antibody detection with CAP survey. Tissue antigens 85: 340.

11. Haas M, Sis B, Racusen LC, Solez K, Glotz D, et al. (2014) Banff 2013 meeting report: inclusion of c4d-negative antibody-mediated rejection and antibody-associated arterial lesions. Haas et al. Am J Transplant 14: 272-283.

12. Lal V, Mishra MN (2015) Luminex crossmatch for pretransplant evaluation of renal transplant recipients. Hum Immunol: 76.

13. Takemoto SK, Zeevi A, Feng S, Colvin RB, Jordan S, et al. (2004) National conference to assess antibody-mediated rejection in solid organ transplantation. Am J Transplant 4: 1033-1041.

14. Billen EV, Voorter CE, Christiaans MH, van den Berg-Loonen EM (2008) Luminex donor-specific crossmatches. Tissue Antigens 71: 507-513.

15. Phayphet M, Alamartine E, Mariat C, Absi L, Berthoux F (2006) Harmful Effect of Anti-Class II Antibodies in Kidney Transplant Patients who Experienced an Acute Rejection Episode. Tx Med 18: 78-82.

16. Grenzi PC, de Marco R, Silva RZ, Campos EF, Gerbase-DeLima M (2006) Antibodies against denatured HLA class II molecules detected in luminex-single antigen assay. Clin Transpl. 201-205.

17. Lynch RJ, Platt JL (2010) Accommodation in renal transplantation: unanswered questions. Curr Opin Organ Transplant 15: 481-485. 